\title{
3-D GLACIER SUBSURFACE CHARACTERIZATION USING SAR POLARIMETRY
}

\author{
Giuseppe Parrella $^{1,2}$, Konstantinos Papathanassiou $^{1}$ and Irena Hajnsek ${ }^{1,2}$ \\ ${ }^{1}$ German Aerospace Center - Microwaves and Radar Institute, Wessling, Germany \\ ${ }^{2}$ ETH Zurich - Institute of Environmental Engineering, Zurich, Switzerland
}

\section{INTRODUCTION}

The paper introduces a new polarimetric scattering model able to interpret and invert coherent polarimetric SAR (PolSAR) measurements over glaciers and ice sheets. Individual scattering components related to ice lenses and pipes are considered to model the subsurface scattering structure of ice sheets. The model is able to interpret the scattering amplitudes, their ratios at the different polarizations as well as the observed polarimetric phase differences. The co-polarization (HH-VV) phase difference is related to the structural anisotropy of the firn layer and can be used to estimate its thickness. The model is validated against L-band PolSAR data acquired by the ESAR sensor of the German Aerospace Center (DLR) over the Austfonna ice cap in Svalbard during the ICESAR2007 campaign and available GPR profiles. L-band GPR profiles measured in spring 2007 by the Norwegian Polar Institute and the University of Oslo are also used to support the data analysis and interpretation.

\section{STATE OF THE ART}

SAR configurations allow the systematic monitoring of glaciers and ice sheets also during the winter time when the snow cover is dry and almost invisible at low frequencies. First attempts to monitor glaciers by means of SAR have been reported back in the 80's [1],[2]. Polarimetric SAR (PolSAR) sensors provide data that allow to assess information about the physical and geometrical properties of scatterers. However, very few studies have been carried out about PolSAR backscattering modelling to gain physical interpretation and extract parameters. A first polarimetric model was presented in [3] to interpret C-, L- and P-band observations of the percolation zone of Greenland. Ice lenses and glands, buried in a shallow layer of dry snow, were assumed as main source of scattering and modelled as horizontally and vertically oriented cylinders, respectively. Polarimetric phase differences were speculated to come from reflection processes within the ice bodies. The agreement between model and PolSAR observables was rather good at C-band but poorer at L- and P-band. A three-component model for L- and P-band PolSAR measurements was developed in [4]. Backscattering was modelled as a sum of a 
surface component generated at a shallow snow-ice interface, a volume contribution from randomly oriented dipoles related to ice grains and inclusions embedded in the underlying ice mass, and a component associated to the presence of sastrugi at the glacier surface. Despite the higher complexity, this model could not explain the polarization phase differences often observed in the SAR data.

\section{POLARIMETRIC SCATTERING MODEL}

The objective of this paper is to introduce a novel model-based polarimetric decomposition in order to gain a better physical interpretation for the specific case of backscattering from the percolation zone of ice masses. Most of the scattering is attributed to solid ice clusters (pipes and lenses) in the upper meters of snow and firn [3]. In fact, ice pipes and lenses typically extend for few tens of cm (comparable to the L-band wavelength) with a thickness of some $\mathrm{cm}$ [3]. Lenses are modelled as horizontally oriented oblate spheroids whereas pipes are described as prolate spheroids mainly aligned to the vertical direction. Thus, different shapes are assumed for different classes of scatterers. In the case of propagation of the radar signal through a dielectrically anisotropic material, differential propagation effects become significant. Of interest for this study is the case of polar firn, which generally characterizes the near-surface layers of glaciers and ice sheets. As shown in [5], the characteristic anisotropic structure of firn can be related to the polarization phase differences observed in SAR data. According to this approach, firn is modelled as a two-phase mixture of vertically elongated ice grains immersed in a background of air to derive the permittivity components in the vertical and horizontal plane. Once the dielectric anisotropy of firn is obtained, differential propagation effects are modelled in terms of the polarimetric covariance matrix elements, which are successively arranged in a propagation matrix $[P]$ and incorporated in the simulated scenario:

$$
\left[C_{\text {percol }}\right]=\left(f_{\text {obl }} \cdot\left[C_{o b l}\right]+f_{\text {pro }} \cdot\left[C_{\text {pro }}\right]\right) \circ[P]+[N]
$$

The terms $\left[C_{o b l}\right]$ and $\left[C_{\text {pro }}\right]$ are the covariance matrices corresponding to the oblate and prolate volume contributions respectively, while $f_{o b l}$ and $f_{\text {pro }}$ are the corresponding intensity factors. Finally, the matrix $[N]$ accounts for an additive noise term and the symbol ' $\circ$ ' represents the element-wise product. The model is completed by accounting for refraction and transmission effects occurring at the air/snow interface at the glacier surface. The decomposition is performed by minimizing a cost function which jointly accounts for the relative errors between modelled and observed polarimetric signatures (entropy, mean alpha angle and HH/VV ratio). In a preliminary step, a thickness estimate of the firn layer is obtained from the co-polarization phase difference [5] assuming an average density and degree of anisotropy of the grains according to the ground measurements and literature values, respectively. The inverted thickness is successively employed to include differential propagation 
effects in the minimization procedure. Other parameters estimated from the polarimetric decomposition are the prolates-to-oblates (pipes-to-lenses) intensity ratio $\left(f_{\text {pro }} / f_{\text {obl }}\right)$ and the orientation angle distribution widths of oblates and prolates.

\section{EXPERIMENTAL DATA AND ANALYSIS}

For a preliminary analysis of the SAR data, covariance matrices have been estimated using a spatial averaging of about 100 effective looks. Four polarimetric signatures are investigated: the co-polarization (co-pol) ratio $\left|\mathrm{S}_{\mathrm{HH}}\right|^{2} /\left|\mathrm{S}_{\mathrm{Vv}}\right|^{2}$, the co-pol phase difference $\phi_{\mathrm{HH}-\mathrm{vv}}$, the scattering entropy $H$ and the mean alpha angle $\alpha$. The co-pol ratio is an indicator of the scatterers orientation: values $>1$ suggest scattering from horizontally oriented structures, $\approx 1$ are attributed to a random volume, and $<1$ indicate scattering from vertically oriented features or from a (slightly rough) surface [6]. The co-pol ratio extracted from the data (Fig. 1(a)) is spatially very homogeneous, with values $>1$, which suggest the presence of horizontal features. The co-pol phase difference provides information about the dielectric properties of the medium. The co-pol phase map for the investigated area (Fig. 1(b)) shows relatively high values. A pronounced increase from $30^{\circ}$ up to $90^{\circ}$ across range suggests the presence of anisotropic firn [5]. The polarimetric entropy indicates whether a dominant scattering mechanism is present (low entropy), or more mechanisms contribute significantly to the total backscatter (high entropy). In the case of volume only scattering, the entropy becomes a measure of the randomness of the orientation distribution of the scatterers within the volume. Similarly to the other polarimetric signatures, the entropy over the test site appears homogeneous across azimuth and with a significant variation across range, rising from 0.4 to 0.8 from near to far range (Fig. 1(c)). The high entropy, together with co-pol ratio $>1$, confirms that volume scattering from horizontal features is significant [6]. The set of polarimetric signatures is completed with the mean alpha angle which provides information about type of the main scattering mechanism. For the alpha angle map extracted from the data (Fig. 1(d)) similar considerations as for the entropy apply. The rather high values (up to $50^{\circ}$ ) clearly indicate that volume scattering is the dominant mechanism in the scene [6]. Beside the polarimetric analysis carried out above, the proposed decomposition technique has been applied to the SAR data with the objective of gaining a physical interpretation of the observed signatures. Decomposition results are presented and discussed in the full paper.

\section{ACKNOWLEDGMENTS}

This work is funded by the Helmholtz Alliance EDA - Remote Sensing and Earth System Dynamics, through the Initiative and Networking Fund of the Helmholtz Association, Germany. The authors would like to thank the University of Oslo and the Norwegian Polar Institute who collected and made available the GPR data. 


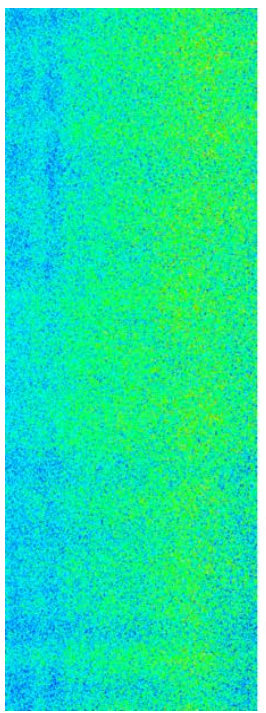

a) $\left|S_{H H}\right|^{2} /\left|S_{V V}\right|^{2}$

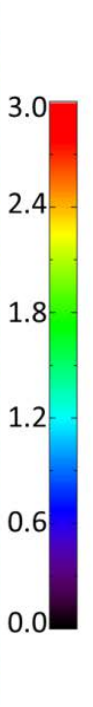

b) $\phi_{\mathrm{HH}-\mathrm{VV}}$

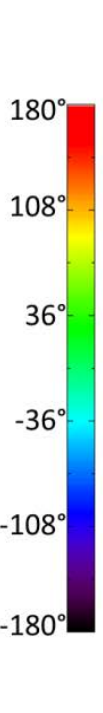

c) $H$
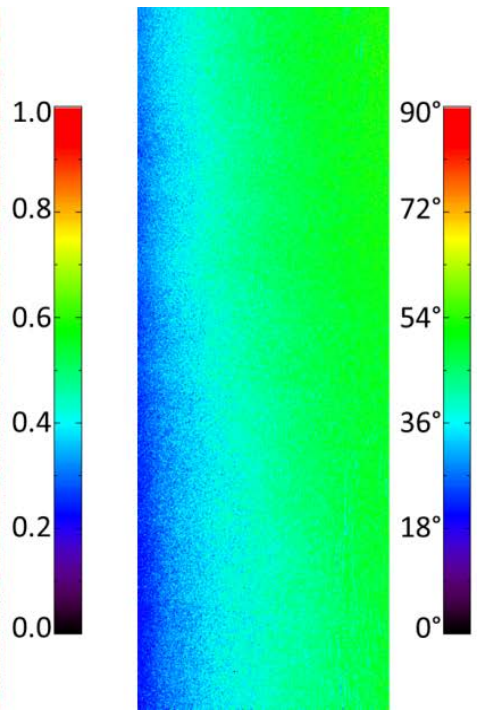

d) $\alpha$

Fig. 1: PolSAR signatures of L-band March North acquisition over the Summit area of Austfonna; a) co-polarization ratio, b) co-polarization phase difference, c) scattering entropy, d) mean alpha angle.

\section{REFERENCES}

[1] H. Rott, “The analysis of backscattering properties from SAR data of mountainous regions,” IEEE J. Oceanic Eng., OE-9(5), 347-355, Dec. 1984.

[2] H. Rott and C. Maetzler, "Possibilities and limits of synthetic aperture radar for snow and glacier surveying," Ann. Glaciol., 9, 1987.

[3] E. J. Rignot, “Backscatter model for the unusual radar echoes from the Greenland ice sheet,” J. Geophys. Res., 100(E5), 9389-9400, May 1995.

[4] J. J. Sharma, et al., "Polarimetric decomposition over glacier ice using long-wavelength airborne PolSAR,” IEEE Trans. Geosci. Remote Sens., 49(1), 519-534, Jan. 2011.

[5] G. Parrella, K. Papathanassiou and I. Hajnsek, "Relating co-polarization phase difference at L-band over land ice to the structure of snow and firn layers,” in Proc. of IGARSS'2014, Quebec City, Canada, 2014, 4840-4843. [6] S. Cloude, Polarisation: Applications in Remote Sensing. London, U.K.: Oxford Univ. Press, 2010. 